\title{
Application of a modified Inception-v3 model in the dynasty-based classification of ancient murals
}

\author{
Jianfang Cao ${ }^{1,2^{*}} \mathbb{D}$, Minmin Yan², Yiming $\mathrm{Jia}^{2}$, Xiaodong $\operatorname{Tian}^{2}$ and Zibang Zhang ${ }^{2}$
}

\author{
* Correspondence: caojianfangcn@ \\ 163.com \\ ${ }^{1}$ Department of Computer Science \\ \& Technology, Xinzhou Teachers \\ University, No. 10 Heping West \\ Street, Xinzhou 034000, China \\ ${ }^{2}$ School of Computer Science \& \\ Technology, Taiyuan University of \\ Science and Technology, Taiyuan \\ 030024, China
}

\begin{abstract}
It is difficult to identify the historical period in which some ancient murals were created because of damage due to artificial and/or natural factors; similarities in content, style, and color among murals; low image resolution; and other reasons. This study proposed a transfer learning-fused Inception-v3 model for dynasty-based classification. First, the model adopted Inception-v3 with frozen fully connected and softmax layers for pretraining over ImageNet. Second, the model fused Inception-v3 with transfer learning for parameter readjustment over small datasets. Third, the corresponding bottleneck files of the mural images were generated, and the deep-level features of the images were extracted. Fourth, the cross-entropy loss function was employed to calculate the loss value at each step of the training, and an algorithm for the adaptive learning rate on the stochastic gradient descent was applied to unify the learning rate. Finally, the updated softmax classifier was utilized for the dynasty-based classification of the images. On the constructed small datasets, the accuracy rate, recall rate, and F1 value of the proposed model were $88.4 \%, 88.36 \%$, and $88.32 \%$, respectively, which exhibited noticeable increases compared with those of typical deep learning models and modified convolutional neural networks. Comparisons of the classification outcomes for the mural dataset with those for other painting datasets and natural image datasets showed that the proposed model achieved stable classification outcomes with a powerful generalization capacity. The training time of the proposed model was only 0.7 $s$, and overfitting seldom occurred.
\end{abstract}

Keywords: Mural classification, Dynasty identification, Transfer learning, Inception-v3 model

\section{Introduction}

Murals are one of the most ancient painting forms in human history and are referred to as "the art on the wall." Ancient murals are important cultural legacies and have significant research value for cultural heritage conservation [1]. China is a country with a long history; a large number of Chinese murals have been preserved, which provides rich resources for mural research. Ancient Chinese murals have undergone numerous historical periods. Based on the time when the murals were created, six historical periods can be identified, i.e., Stone Age, Qin/Han dynasties, Wei/Jin dynasties, Sui/Tang

(c) The Author(s). 2021 Open Access This article is licensed under a Creative Commons Attribution 4.0 International License, which permits use, sharing, adaptation, distribution and reproduction in any medium or format, as long as you give appropriate credit to the original author(s) and the source, provide a link to the Creative Commons licence, and indicate if changes were made. The images or other third party material in this article are included in the article's Creative Commons licence, unless indicated otherwise in a credit line to the material. If material is not included in the article's Creative Commons licence and your intended use is not permitted by statutory regulation or exceeds the permitted use, you will need to obtain permission directly from the copyright holder. To view a copy of this licence, visit http://creativecommons.org/licenses/by/4.0/. 
dynasties, Song/Jin dynasties, and Ming/Qing dynasties. Traditionally, mural dynasty classification is performed using a visual method in combination with preserved documents. However, due to the age of some murals, the similarities in content among murals and/or severe damage, dynasty recognition for some murals cannot be completed, and therefore, more rapid and scientific dynasty classification methods for ancient murals are needed.

Digital protection and restoration and structural reconstruction are helpful tools for the permanent preservation of mural information [2]. They allow infinite replications of an image and realize its virtual exhibition by constructing a high-quality, original, and complete data file for future generations to appreciate. Awareness of the dynasty when a mural was created helps visitors better understand the hidden culture of the mural. In 2003, Pan et al. [3] proposed a set of intelligent techniques and realized the digital repair of murals. Yuan et al. [4] used the nearest neighbor method for digital repair of ancient paintings with tearing and shedding. Wang et al. [5] explored the texture and structure of Dunhuang murals using the sparse modeling method. The methods applied in these studies have significance in researching the dynasty classification of ancient murals.

From text-based image retrieval to content-based image retrieval, the analysis of image features, such as texture and color, has a crucial role in image retrieval and classification. Among various methods for content-based image retrieval, Zhang et al. [6] proposed an algorithm in which tree-structured data are transformed into a vectorial representation. According to this algorithm, a Tree2Vector framework is proposed: a two-level tree is constructed to extract both the overall features and local features of an image, which are then transformed into vectorial representations and coded for image retrieval. Their results showed the efficacy of the proposed method. To date, numerous studies of mural classification based on traditional methods, such as feature extraction and classifier-based classification, have been reported. Tang et al. [7] proposed a grouping multi-instance learning method for optimizing the latent support vector machine (SVM) model, targeting the issues of the intraclass difference between mural images and the noise contained in the images. Based on the composition and features of ancient murals, Wang et al. [8] proposed a model in which the relevance between the composition of an ancient mural and its semantics was integrated. Tang et al. [9] utilized extracted contour features to measure the similarity degree between two mural images. Although traditional methods can realize the extraction of certain features from murals, they suffer from insufficient generalization capacities in terms of feature extraction and classification outcomes due to the diverse content of murals. Wang et al. [10] proposed a new convolutional neural network $(\mathrm{CNN})$ for classifying multispectral images, which reduced the influence of calculation errors on the precision of mural pigment recognition. With the continuous development of technology for mural digitalization and image data classification, scholars have proposed various strategies for mural classification. Sun [11] investigated the visual features and perception of the Taoist murals in the Ming/Qing dynasties located in northern Shananxi, based on which he classified the image data of the murals into the categories of correlated images, reference images, and associated images. Hao [12] employed the target detection method to position the image characters of the investigated murals according to the special artistic styles of character mural images created in different historical periods; 
they extracted the features associated with the appearance, headdress, and costumes of the mural characters for mural classification. These scholars have made useful attempts at mural classification based on different methods and from different perspectives. However, research on methods for mural dynasty recognition has rarely been reported.

As deep learning techniques continuously develop, CNNs have proven effective in the fields of image recognition and classification [13-15]. In recent years, CNNs have been gradually applied in the repair, superresolution reconstruction, and classification of ancient mural images. Li et al. [16] encoded painting styles with a CNN for 194 Dunhuang Grotto murals, whose time of creation had been identified, and then utilized color descriptors for color vision code extraction. Zou et al. [17] extracted and encoded the appearance and formed features of mural images with a scale-invariant feature transform (SIFT) and kAS descriptors; then, they applied a supervised learning strategy in combination with a feature histogram for mural dynasty classification. Although these methods can achieve high classification accuracy, their final outcomes for some mural images are inconsistent with the officially identified dynasties due to the small number and identical source of the investigated murals as well as great similarities among murals in terms of content and painting styles.

Inception-v3 [18], which was proposed by the Google team, is a network architecture based on modifications of AlexNet. Inception-v3 has been frequently applied in image recognition. Liu et al. [19] combined Inception-v3 with S-Mask R-CNN and fused transfer learning in the input layer. They applied this technique in lesion recognition for prostate images in the to-be-detected region determined by S-Mask R-CNN; their method achieved higher canceration identification results than traditional artificial recognition methods. Chowdary et al. [20] fused Inception-v3, VGG19, Resnet50, and MobileNet for facial emotion recognition of 918 images in the CK+ dataset. The results showed that the fusion between transfer learning and Inception-v3 achieved high classification accuracy. Enlightened by the notion of convolution decomposition in the Inception-v3 model, Yeh et al. [21] decomposed the convolution of the extended layer in a lightweight network. This operation reduced the calculation complexity of the network and guaranteed the stability of the feature expressiveness of the model to effectively extract image features from multiple scales. All these studies have proven the advantages of Inception-v3 in image recognition and target detection and recognition. Compared with Inception-v1 and Inception-v2, Inception-v3 integrates convolution decomposition to accelerate the calculation process; it possesses a deeper network architecture, with an input size of $299 \times 299$. In addition, this model requires fewer parameters and shorter training times. Based on these virtues of Inception-v3, this study proposed a transfer learning-fused Inception-v3 model to complete ancient mural dynasty identification. The mural images for testing the classification performance of the proposed model reflected different dynasties and varying painting styles.

This study is organized as follows: In the following section, we introduced the features and detailed network configuration of the Inception-v3 model as well as the transfer learning-fused ancient mural dynasty identification model that was proposed in this study. In the third section, we described the sources and detailed data of the datasets selected for the experiment in this study and described the main results. In the fourth section, we drew conclusions based on the main findings obtained in this study and suggested a direction for future research. 


\section{Methods}

\subsection{Inception-v3 model}

The Inception model is a deep CNN architecture that was proposed by Szegedy et al. in the Large-Scale ImageNet Visual Identification Challenge 2014; its aim was to weaken the influence of computational efficiency and low parameters in application situations [17]. The image size input into Inception-v3 was $299 \times 299$. Although this size is $78 \%$ larger than that of VGGNet $(244 \times 244)$, the running speed of Inception-v3 is faster than that of VGGNet. The main reasons for this high efficiency of Inception-v3 are presented as follows: compared with AlexNet, the number of parameters of Inception-v3 is fewer than half that of AlexNet $(60,000,000)$ and fewer than one fourth that of VGGNet $(140,000,000)$; additionally, the number of floating-point computations of the whole Inception-v3 network is approximately 5,000,000,000, which is much larger than that of Inception-v1 (approximately 1,500,000,000). These characteristics make Inception-v3 more practical, that is, it can be easily implemented in a common server to provide a rapid response service.

Inception-v3 adopts convolutional kernels of different sizes, which enables it to own receptive fields of different areas. To reduce the design space of the network, it adopts a modular system followed by final joining, thereby realizing the fusion of features of varying scales. The network parameters of Inception-v3 are summarized in Table 1. The configuration of the Inception-v3 model is shown in Fig. 1.

In Inception-v3, a batch normalization $(\mathrm{BN})$ layer is inserted as a regularizer between the auxiliary classifier and the fully connected (FC) layer. In the BN model, the batch gradient descent method can be employed to accelerate the training speed and model convergence of the deep neural network. The formulas of BN are expressed as follows:

$$
\begin{gathered}
\mathrm{B}=\left\{X_{1 \ldots m}\right\}, \gamma, \beta \\
\left\{y_{i}=B N_{\gamma, \beta}\left(\chi_{i}\right)\right\} \\
\mu_{\mathrm{B}} \leftarrow \frac{1}{m} \sum_{i=1}^{m} X_{i}
\end{gathered}
$$

Table 1 Network structure of the Inception-v3 model

\begin{tabular}{lll}
\hline Type & Patch size/stride & Input size \\
\hline conv & $3 \times 3 / 2$ & $299 \times 299 \times 3$ \\
conv & $3 \times 3 / 1$ & $149 \times 149 \times 32$ \\
conv & $3 \times 3 / 1$ & $147 \times 147 \times 32$ \\
pool & $3 \times 3 / 2$ & $147 \times 147 \times 64$ \\
conv & $3 \times 3 / 1$ & $73 \times 73 \times 64$ \\
conv & $3 \times 3 / 2$ & $71 \times 71 \times 80$ \\
conv & $3 \times 3 / 1$ & $35 \times 35 \times 192$ \\
$3 \times$ Inception & -- & $35 \times 35 \times 288$ \\
$5 \times$ Inception & -- & $17 \times 17 \times 768$ \\
$2 \times$ Inception & -- & $8 \times 8 \times 1280$ \\
pool & $8 \times 8$ & $8 \times 8 \times 2048$ \\
linear & logits & $1 \times 1 \times 2048$ \\
softmax & classifier & $1 \times 1 \times 1000$ \\
\hline
\end{tabular}




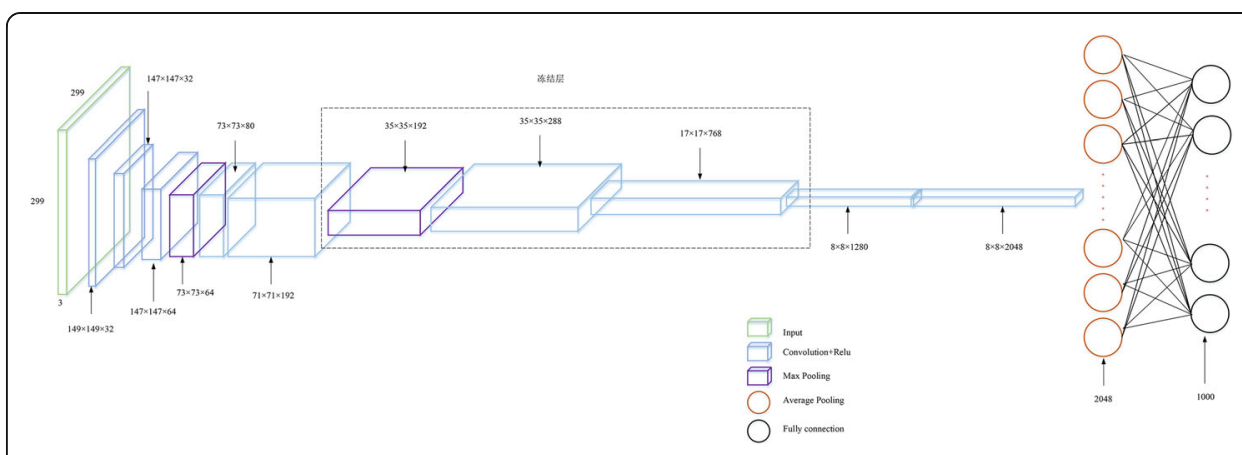

Fig. 1 Structure diagram of the Inception-v3 model

$$
\begin{aligned}
& \sigma_{\mathrm{B}}{ }^{2} \leftarrow \frac{1}{m} \sum_{i=1}^{m}\left(X_{i}-\mu_{\mathrm{B}}\right)^{2} \\
& \hat{X}_{i} \leftarrow \frac{X_{i}-\mu_{\mathrm{B}}}{\sqrt{\sigma_{\mathrm{B}}^{2}+\varepsilon}} \\
& y_{i} \leftarrow \gamma \hat{X}_{i}+\beta=B N_{\gamma, \beta}\left(X_{i}\right)
\end{aligned}
$$

where $x$ is the minimum activation value of batch $B, m$ is the number of activation values, $\gamma$ and $\beta$ are learnable parameters $(\gamma$ is responsible for adjusting the variance in the value distribution and $\beta$ is responsible for adjusting the position of the average value), $\mu_{\mathrm{B}}$ represents the average value in one dimension, $\sigma_{\mathrm{B}}{ }^{2}$ is the standard deviation in each dimension of the feature map, and $\varepsilon$ is a constant.

Furthermore, in Inception-v3, large convolution kernels are divided into small convolution kernels in series, convolution and pooling are connected in parallel, and LSR labels are added for regularization based on the smoothing criteria. In addition, considering the distribution inconsistency between inputs and outputs in a traditional deep neural network, which creates great obstacles for feature extraction, BN is introduced into Inception-v3. By normalizing the input into each layer, the learning effect is optimized.

\subsection{Transfer learning-fused model for ancient mural dynasty recognition}

Research on mural images suffers from issues such as low quality, a small number of murals and collection difficulty. To deeply extract image features over small mural datasets, the model proposed in this study was pretrained over the large dataset ImageNet, and then the knowledge obtained through transfer learning was applied to small mural datasets to achieve dynasty recognition and classification for the murals.

\subsubsection{Transfer learning-fused model for dynasty-based classification}

In this study, the model proposed for dynasty-based classification of ancient murals consists of the feature extraction section and classification section. For feature extraction, a CNN is adopted. The classification section is composed of FC layers and softmax layers and is illustrated in Fig. 2.

As shown in Fig. 2, in the proposed model, the convolution layers and pooling layers before the FN layer and softmax layer are frozen. The size of the convolution kernel of each convolution layer is $3 \times 3$. The size of the first pooling layer is $3 \times 3$ and that of 


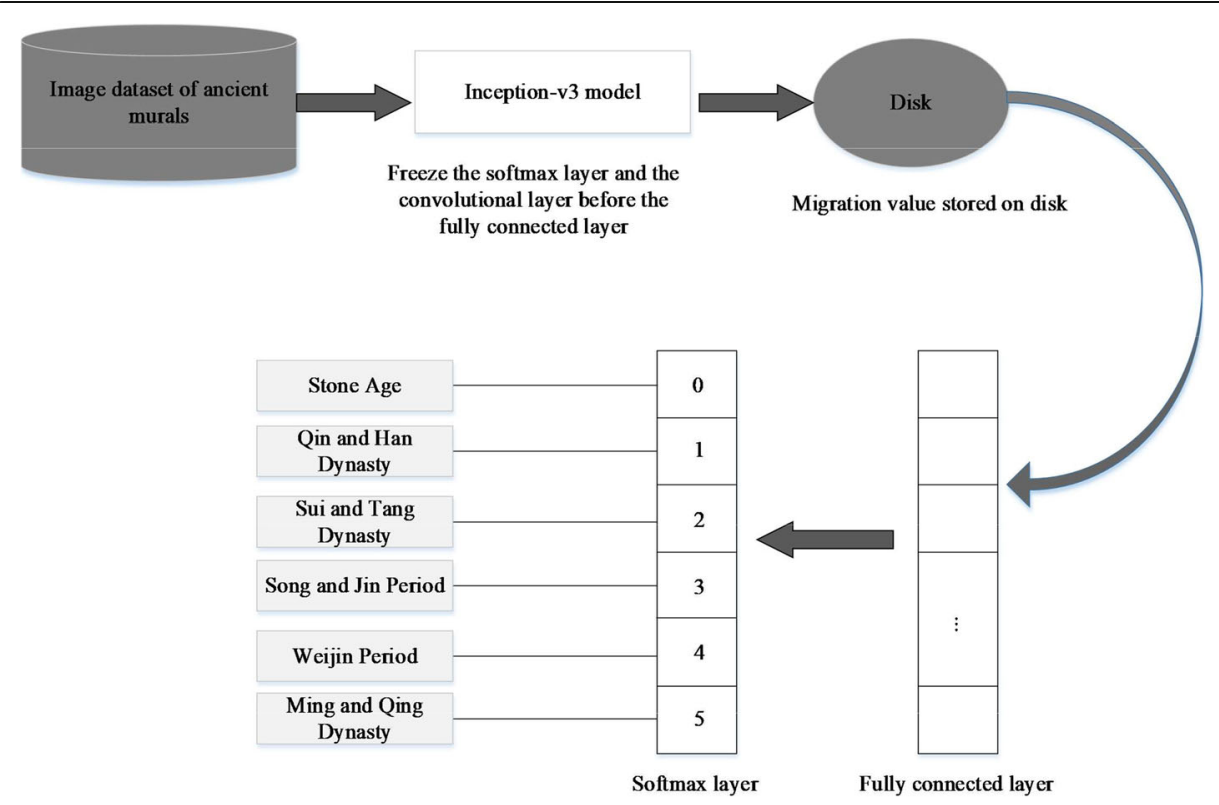

Fig. 2 Dynasty-based classification model for ancient murals

the second is $8 \times 8$. The convolution layers and pooling layers are responsible for extracting low-level features. The inception structure in the middle consists of three module groups: the size of the image input into the first group is $35 \times 35 \times 288$; in the second group, the size of the feature map is reduced to $17 \times 17 \times 768$ and filters are added; in the third group, the size of the image is $8 \times 8 \times 1,280$, and the parallel convolution and pooling structure is adopted. The Inception-v3 model with frozen convolution and pooling layers is pretrained over the large dataset ImageNet. Afterward, the last two layers, i.e., the FC layer and softmax layer, are trained over mural datasets. The convolution layer before the FC layer and softmax layer is the bottleneck layer, which is used to calculate the bottleneck values for mural images during training. In this layer, the extracted features are transformed into a 2048-dimensional eigenvector. The position for bottleneck features is the feature output position, and the generated eigenvector is applied as the output node in the softmax layer. The knowledge obtained from deep learning is stored in the disc, and the deep-level features of ancient mural images are extracted.

\subsubsection{Modifications}

\section{(1) Fusion with transfer learning}

The challenges associated with existing ancient murals are that they are small in number, of low quality, and often severely damaged, which create difficulties in image collection and screening. In addition, for the purpose of dynasty-based classification, mural images of different historical periods should be collected. However, the current technique for mural image classification remains immature, and for some murals, the 
dynasties in which they were created have not been identified. This condition makes the task of large data collection more difficult.

To further enhance the learning efficiency of the model and to better extract the deep-level features of murals, transfer learning was fused into the Inception-v3 model in this study. Transfer learning is a type of machine learning in which common knowledge obtained from learning is used to solve problems in other fields; its aim is to accomplish the transfer of valuable information learned in some fields to other fields. Transfer learning can improve the stability and generalization of the model, thereby avoiding the negative influence of the changes in image pixels on the final classification outcomes.

Specifically, transfer learning was utilized in this study as follows. The Inception-v3 model was pretrained over ImageNet, and the low-level image features were extracted. The knowledge obtained from transfer learning as the output of the bottleneck layer was applied over ancient mural datasets. The convolution layers before the FC and softmax layers were frozen. By continuously adjusting the parameters of the network, a new FC layer and a new softmax layer were trained for extracting the deep-level features of the mural images, thus realizing model training and image classification in a relatively short time period.

(2) Algorithm for the adaptive learning rate on stochastic gradient descent (ALR-SGD)

In the SGD algorithm, the loss functions are stochastically selected over some training data during each iteration for gradient descent optimization to accelerate the parameter update speed. The ALR-SGD algorithm enables a consistent learning rate to be applied during the whole experiment.

To maintain the convergence rate of the model during training, an FC layer was used at the end of the model proposed in this study. This layer outputs the score of each possible dynasty when the mural was created and then calculates the distribution distance between the predicted dynasty classification of the image according to the proposed model and the authentic dynasty to which the image belongs. In addition, a cross-entropy function was employed as the loss function in this study to optimize the errors of the model during backpropagation.

If the learning rate is low, the convergence rate of the parameters with a large gradient will gradually decrease. If the learning rate is high, the parameters that have been optimized may become unstable. To solve the problem that some parameters near the minimum value have a large gradient, in this study, the ALR-SGD algorithm was utilized as the optimizer of the proposed model, the cross-entropy function was applied as the minimized cost function, and the learning rate was properly modified to achieve better convergence to avoid saturating the learning rate during model training.

(3) Cross-entropy loss function

To solve the problem of gradient disappearance and to assess the difference between the authentic value and the predicted value, the cross-entropy function and the softmax function were combined into the loss function in this study. A lower cross-entropy value indicates a smaller difference between the actual output and the expected 
outcome and a better effect. Given the same conditions, the cross-entropy function has a faster learning rate than a quadric cost function, and compared with the sigmoid function, it can avoid a decrease in the learning rate.

The cross-entropy function equation is expressed as follows:

$$
H(y, p)=-\sum_{i} y_{i}^{\prime} \log \left(y_{i}\right)
$$

where $y$ is the predicted value, $y_{i}^{\prime}$ is the actual value, and $i$ is the number of categories.

Cross-entropy represents the distance between the actual output and the expected output. During backpropagation, the larger the error between the actual value and the predicted value is, the larger the conditioned amplitude of the parameter and the faster the convergence of the model. The cross-entropy value during training that is output at the end of the experiment can be used to determine whether overfitting occurs in the model. In this study, the cross-entropy function and the softmax classifier were combined. The final output of the softmax classifier underwent processing, and crossentropy was used to calculate the loss.

\subsubsection{Flowsheet for mural classification}

The framework of the transfer learning-fused Inception-v3 model for the dynasty-based classification of ancient murals is shown in Fig. 3, which realized the classification task through four primary stages.

\subsubsection{Stage 1: Image preprocessing Input: Ancient mural image datasets;}

Output: Training, testing and verifying datasets;

Step 1: Modify the size of each mural image in the datasets to a pixel at $299 \times 299$;

Step 2: Enlarge the datasets through image reversion, color dithering and lightness enhancement using the data enhancement method;

Step 3: Obtain the expanded datasets;

Step 4: Divide the images in the expanded datasets with the random function: $80 \%$ of the images constitute the training set, $10 \%$ of the images constitute the testing set, and $10 \%$ of the images compose the verifying set.

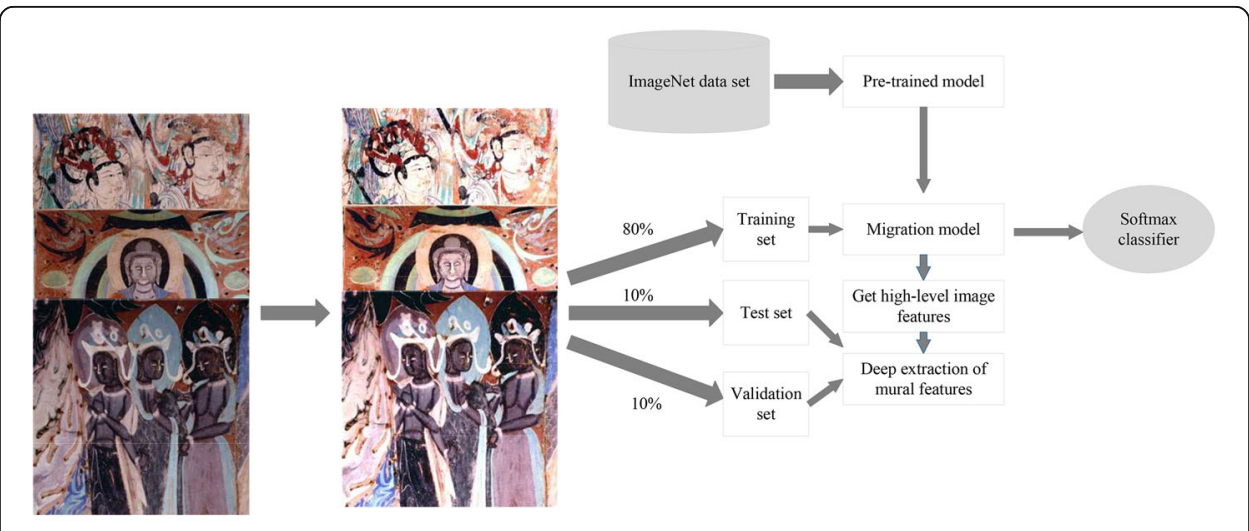

The mural image is processed by data enhancement

Fig. 3 Framework of the Inception-v3 model fused with transfer learning for ancient mural classification 


\subsubsection{Stage 2: Model pretraining Input: Training dataset;}

Output: Trained dataset;

Step 1: Train the downloaded Inception-v3 model over ImageNet;

Step 2: Use the images in the training set for model training to obtain the trained Inception-v3 model;

Step 3: Set the learning rate and batch value and change the number of iterations; record the accuracies at different learning rates and batch values and after different numbers of iterations;

Step 4: Obtain the migration model.

\subsubsection{Stage 3: Model testing Input: Testing dataset;}

Output: Testing accuracy;

Step 1: Introduce the testing dataset into the pretrained migration model;

Step 2: Statistically calculate the classification outcomes to obtain the final accuracy.

\subsubsection{Stage 4: Model verification Input: Verifying dataset;}

Output: Verify the mural image classification accuracy;

Step 1: Introduce the verifying dataset into the pretrained migration model;

Step 2: Statistically calculate the verification outcomes.

\section{Results and discussion}

\subsection{Experimental environment}

The hardware environment primarily consists of an Intel Core i5-8250U CPU, with 16 GB memory and an NVIDIA GeForce MX150 video card. The software environment is Python 3.7 for language programming on the Windows 10 system, with TensorFlow as the framework and PyCharm 2019.3.5 × 64 for compiling.

\subsection{Datasets and sources}

The mural images collected in this study were obtained from the electronic books Underground gallery-brick frescoes of the Wei and Jin Dynasties in Jiayuguan, Complete works of Dunhuang frescoes in China, and Murals of the Song Dynasty in Kaihua Temple. The content of the murals includes Buddha and Bodhisattva images, Buddhist stories, Jataka stories, sutra tales, and various routine production and life activities, such as mulberry picking, carriages and horses, patrolling, cooking, productive laboring, servants, wining and dining, hunting, breeding and grazing, butchering, houses and music playing, which represent the mural arts in different historical periods. Based on clipping and analysis, the collected mural images were divided into the Stone Age, Qin/Han dynasties, Sui/Tang dynasties, Song/Jin dynasties, Wei/Jin dynasties, and Ming/Qing Dynasties. Some examples of different dynasties are shown in Fig. 4, and the mural numbers of different dynasties collected in this study are provided in Table 2.

To enlarge the datasets, data enhancement methods were used to preprocess the murals (some images after preprocessing are shown in Fig. 5). The methods included (1) brightness increase; (2) left and right flipping; (3) random color dithering, including contrast enhancement and color enhancement; and (4) image rotation with a rotation angle of $20^{\circ}$. 
A total of 9700 images were collected in this study, which included enhanced images. These images were divided into the training set, testing set, and verification set at a ratio of 8:1:1. The number of images in different dynasties is summarized in Table 3.

\subsection{Outcomes of model training and verification}

In this study, the training step frequency and experimental learning rate were 10,000 and 0.1, respectively, based on multiple training and testing cycles. Figure 6 shows the variations in the accuracy rate and cross-entropy value during training. During model training, the accuracy rate constantly increased. After the step frequency reached 8000 , the accuracy rate became stable with an accuracy rate of approximately $88 \%$ (Fig. 6a). The cross-entropy loss value continuously decreased as the training proceeded. At a step frequency of 3000, the value became stable and gradually neared 0 (Fig. 6b). During the verification process, the verification accuracy continuously increased, and when the step frequency reached 6000 , the accuracy rate tended to stabilize. The verification cross-entropy loss value showed a continuous decrease followed by a tendency for stability as the verification process proceeded (Fig. 6c, d). Figure 7 shows that when the learning rate was 0.1 , the model achieved the best performance in terms of accuracy. Based on these results, the model proposed in this study showed satisfactory performance and rarely caused overfitting.

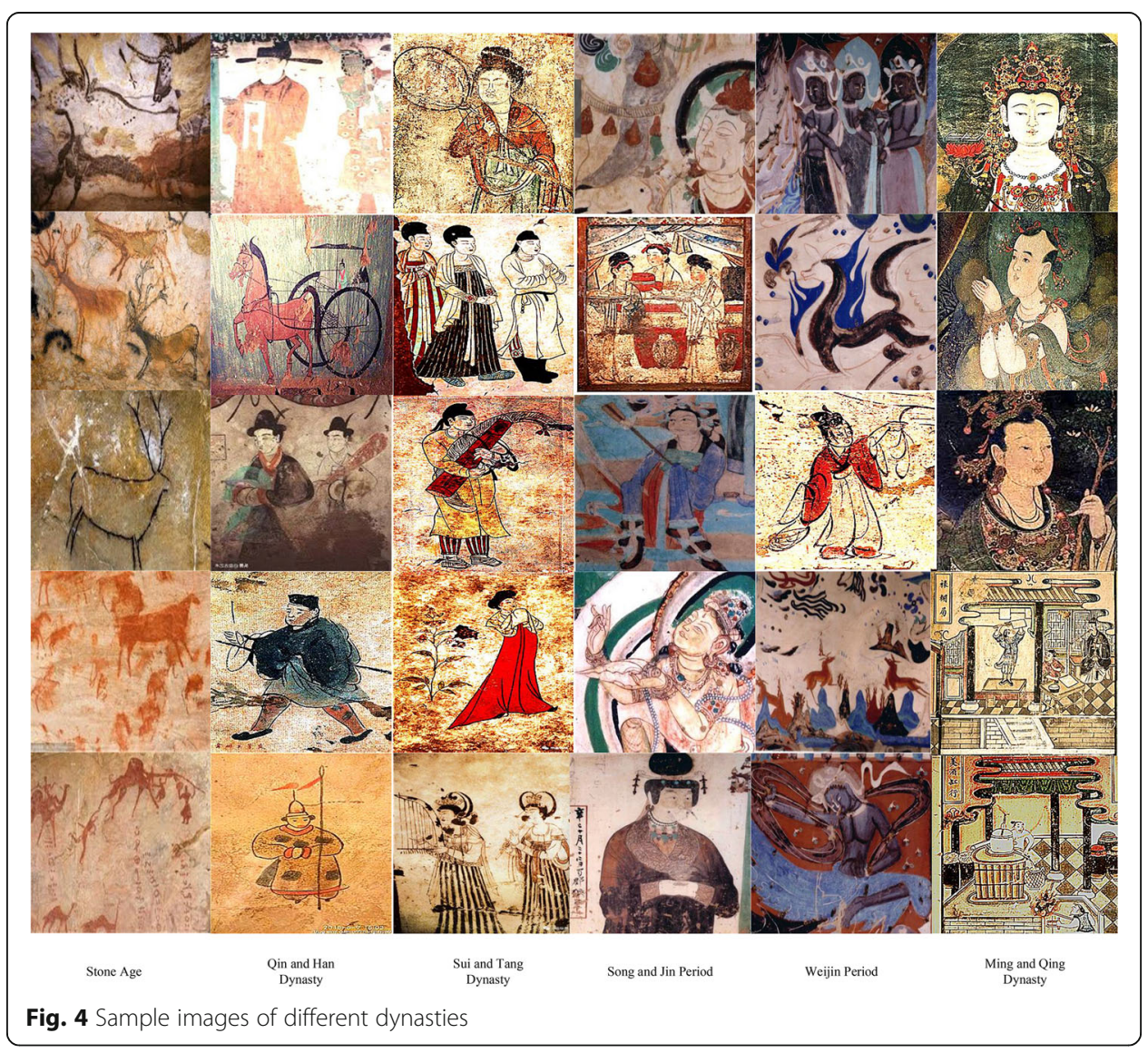


Table 2 Number of murals in each dynasty

\begin{tabular}{ll}
\hline Dynasty type & Number of images \\
\hline Stone Age & 168 \\
Qin/Han Dynasties & 312 \\
Sui/Tang Dynasties & 372 \\
Song/Jin Period & 256 \\
Wei/Jin Period & 436 \\
Ming/Qing Dynasties & 396 \\
Total & 1940 \\
\hline
\end{tabular}

The learning rate serves as a scaling factor for weight adjustment during training. A learning rate that is too large will cause model volatility and convergence failure, whereas a learning rate that is too small can cause model convergence to be too slow, thereby wasting time and calculation resources [22]. In this study, at the same iteration step $(10,000)$, the learning rate was set to $0.001,0.01$, and 0.1 for a multigroup experiment. The variations in the accuracy rate at different learning rates during training are shown in Fig. 7.

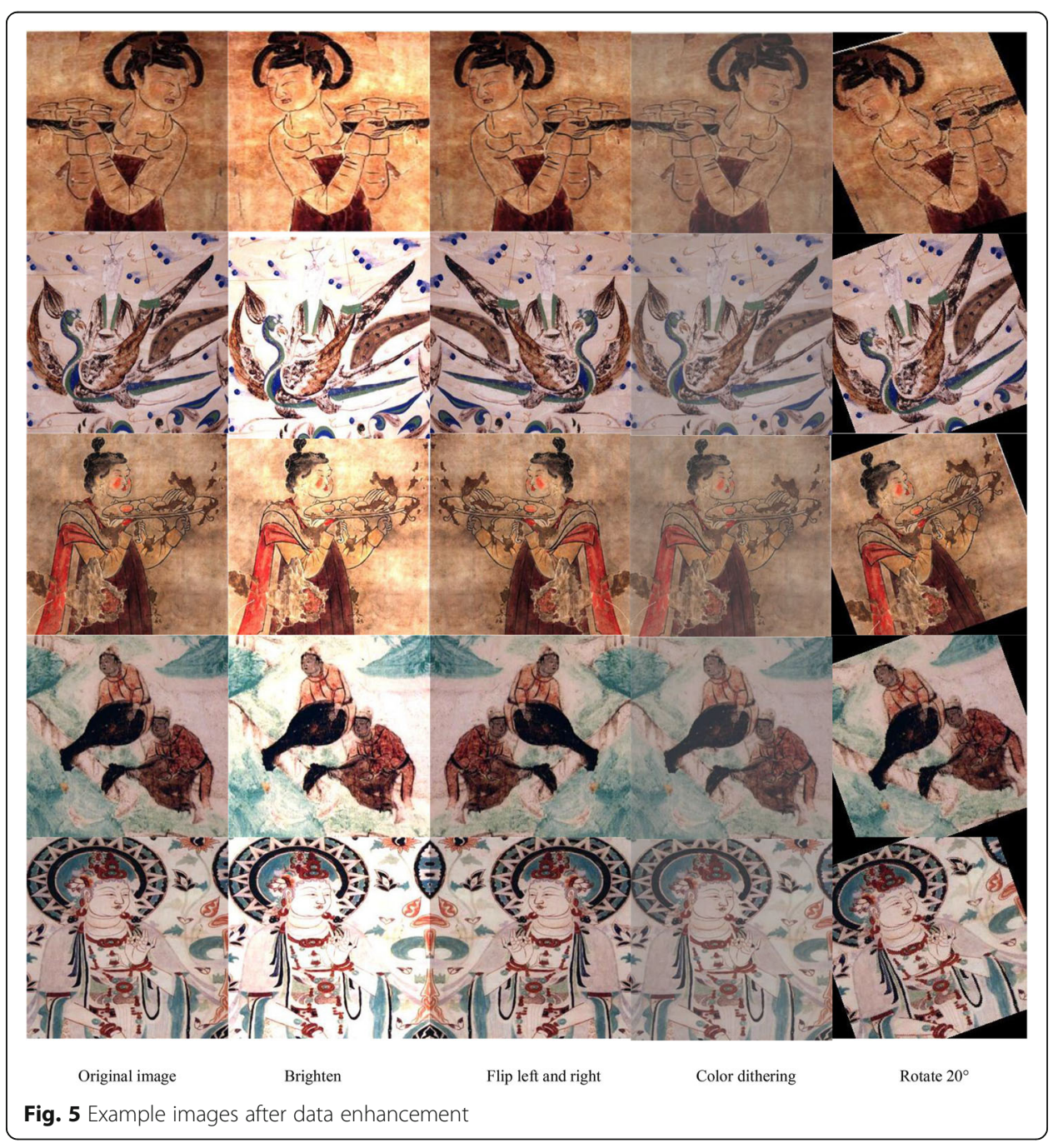


Table 3 Number of mural images in different dynasties

\begin{tabular}{lllll}
\hline Dynasty type & Number of images & Training set & Validation set & Test set \\
\hline Stone Age & 840 & 672 & 84 & 84 \\
Qin/Han Dynasties & 1560 & 1248 & 156 & 156 \\
Sui/Tang Dynasties & 1860 & 1488 & 186 & 186 \\
Song/Jin Period & 1280 & 1024 & 128 & 128 \\
Wei/Jin Period & 2180 & 1744 & 218 & 218 \\
Ming/Qing Dynasties & 1980 & 1584 & 198 & 198 \\
\hline
\end{tabular}

As shown in Fig. 7, the model showed the best performance when the learning rate was 0.1 , with a final maximum accuracy rate of $88.4 \%$. This accuracy rate was $13.1 \%$ and $4.4 \%$ higher than that of the 0.001 group and the 0.01 group, respectively.

During model training, model parameter selection is vital to the occurrence of overfitting and training time. The size of a database matters in the selection of model parameters. After a substantial number of tests, we set the epoch value of the model to 32, batch size to 100, and dropout to 0.5. In addition, we selected Adam as the optimizer and the cross-entropy function as the loss function. To demonstrate the feasibility of the model parameters in this study, we performed tests with parameters at different values; the outcomes are summarized in Table 4.

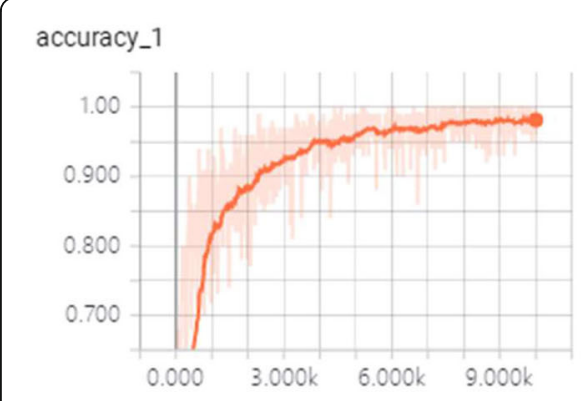

(a) Training accuracy

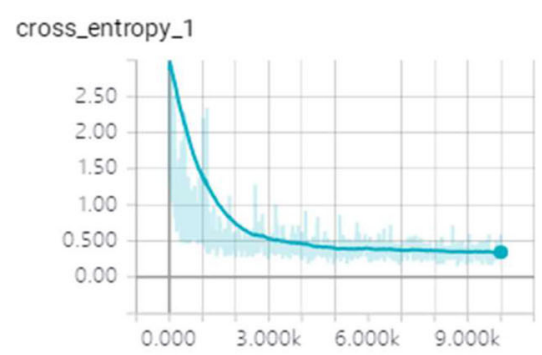

(c) Verification accuracy

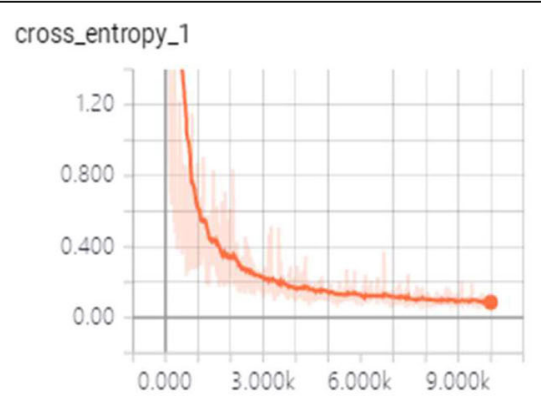

(b) Training cross entropy loss

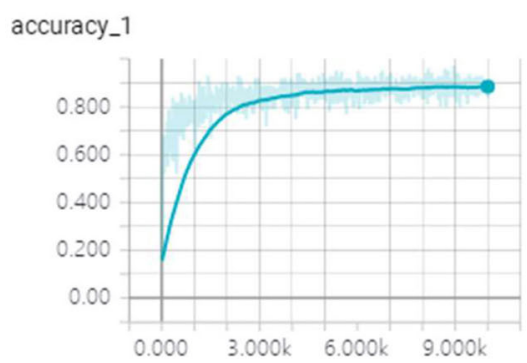

(d) Verification of cross entropy loss

Fig. 6 Results of the accuracy and cross-entropy loss during training and verification. a Accuracy during training. $\mathbf{b}$ Cross-entropy loss during training. c Accuracy during verification. $\mathbf{d}$ Cross-entropy loss during verification 


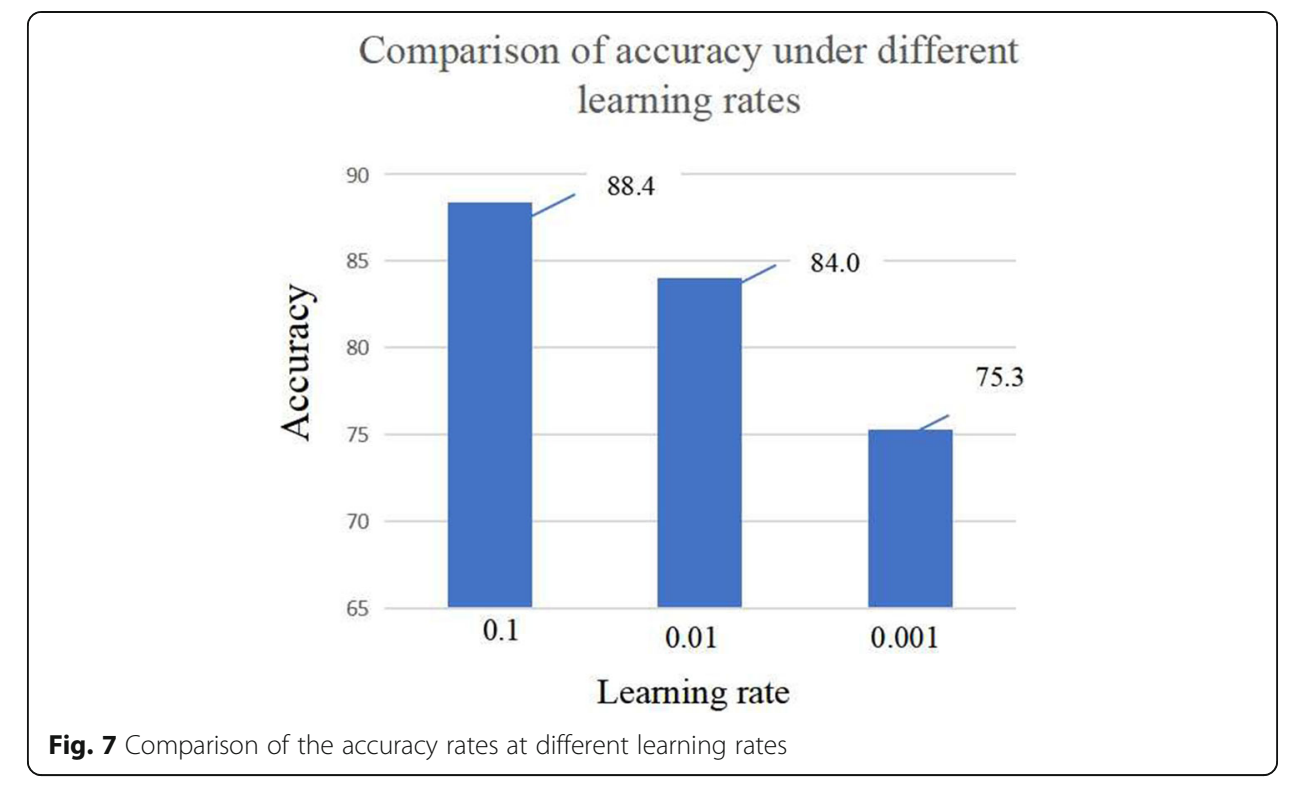

\subsection{Comparisons with other models}

To better illustrate the superiority of the proposed model in dynasty recognition for ancient murals, its accuracy rate was compared with the accuracy rates of typical deep learning network models, which included AlexNet [23], LeNet [24] and VGG [25] and DenseNet [26], MobileNet [27], and modified CNN models, which included LeNet-5 [28], Alex-10 [29], R-VGGNet [30], and Du et al.'s model [31].

(1) Running step number of 10,000 at a learning rate of 0.1

The running time and accuracy rate of the proposed model were compared with those of AlexNet, LeNet, VGG, LeNet-5, Alex-10, R-VGGNet, and AlexNet-S6; the comparison results are shown in Figs. 8 and 9.

As shown in Fig. 8, it only took $0.7 \mathrm{~h}$ for the proposed model to run 10,000 steps, which was 7/25, 7/40, and 7/50 of the time needed for AlexNet, ResNet, and VGGNet, respectively. Compared with the considered modified CNNs, the proposed model also showed great advantages in terms of running time. The following reasons may explain

Table 4 Comparison outcomes for different parameter values

\begin{tabular}{lll}
\hline & Size & Accuracy \\
\hline Number of categories & 6 & - \\
Dropout & 0.5 & - \\
Batch size & 50 & $76.8 \%$ \\
& 100 & $88.4 \%$ \\
Epoch & 16 & 69.4 \\
& 32 & $88.4 \%$ \\
Iterations & 64 & $78.3 \%$ \\
& 5000 & $62.5 \%$ \\
& 10,000 & $88.4 \%$ \\
\hline
\end{tabular}


these differences: AlexNet, ResNet, and VGGNet have deeper network architectures than the proposed model, and therefore, they consume a larger amount of memory space. When the number of calculations, such as the feature extraction for the mural datasets in this study, is too large, they consume a substantial amount of time. In contrast, the advantage of the inception structure is manifested by its convolution decomposition. Given the same size of the perception field, this advantage increases the calculation efficiency of Inception, reduces its number of parameters, and increases its convergence rate. With an increase in the number of extracted features, the training of Inception gradually accelerates, which achieves a balance between the width of the network and its depth, thereby reducing the possibility of overfitting during training.

As shown in Fig. 9, after 10,000 running steps, the accuracy rate of the proposed model increased by $66.1 \%, 19.1 \%, 42.4 \%, 5.4 \%, 13.4 \%, 9.8 \%$, and $4.4 \%$ compared with AlexNet, ResNet, VGGNet, LeNet-5, Alex-10, R-VGGNet, and AlexNet-S6, respectively. Presumably, the modified CNNs (i.e., LeNet-5, Alex-10, R-VGGNet, and AlexNet-S6) reported in the literature are the models that are modified over their corresponding datasets, and thus, they only serve specific purposes. When they were applied to mural datasets, problems such as inappropriate parameters and insufficient feature extraction occurred. In contrast, the model proposed in this study was infused with transfer learning and was trained over the mural datasets after pretraining. Furthermore, during the training process, the parameters of this model were continuously adjusted according to the outcomes of each training session. Therefore, it achieved a higher recognition accuracy rate and could deeply extract the mural features, such as color, texture, and painting styles.

(2) With the same running time

Over the mural datasets constructed in this study, the running time was set to $1 \mathrm{~h}$, and the accuracy, recall rate, and F1 value of the proposed model were compared with those of LeNet-5, Alex-10, R-VGGNet, and AlexNet-S6. The results are shown in Fig. 10.

After a 1-h running time, the accuracy rate, recall rate, and F1 value of the proposed model were $88.4 \%, 88.36 \%$, and $88.32 \%$, respectively, which increased by more than $8 \%$

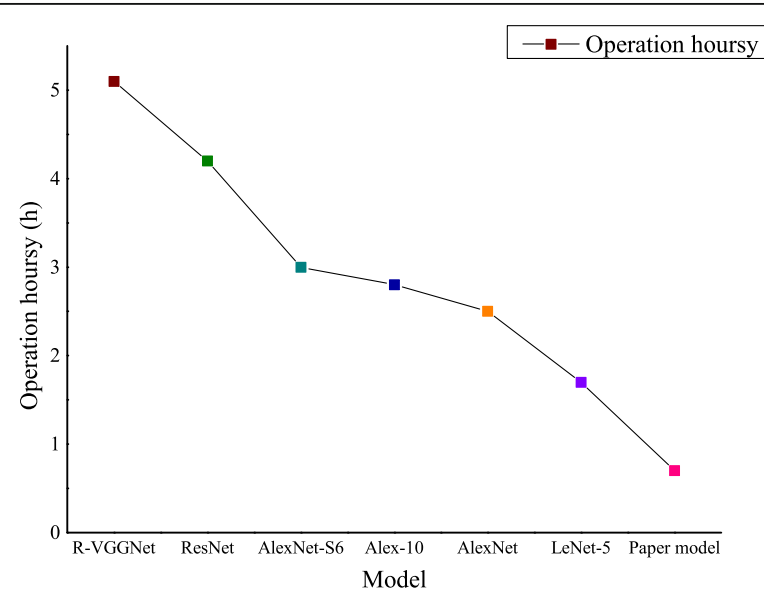

Fig. 8 Comparison of the running times of different models 


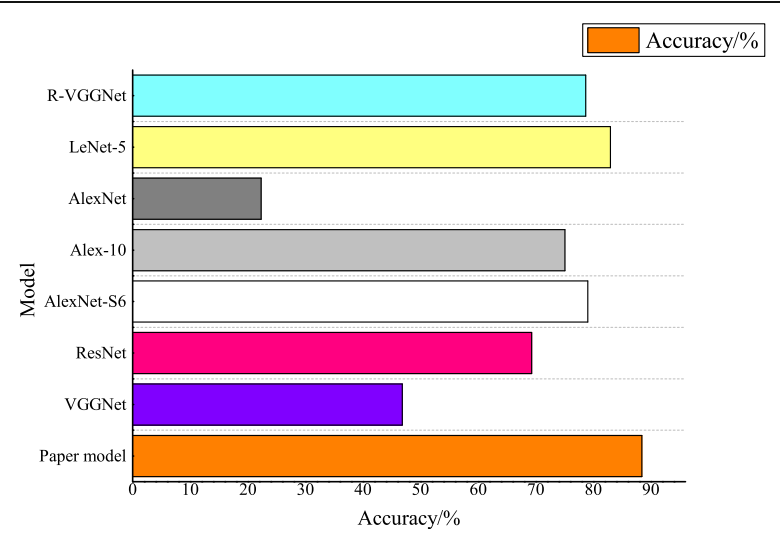

Fig. 9 Comparison of the accuracies of different models

in terms of each index compared with any of the deep learning CNNs (i.e., LeNet-5, Alex-10, R-VGGNet, and AlexNet-S6). The primary reason for these increases is that the model proposed in this study was subjected to fine adjustment according to the features specifically owned by murals, and the network parameters were more suitable for the feature extraction of the datasets employed in this study. In contrast, other network models failed to extract the abundant features of the murals after a 1-h running time due to the large number of parameters and the long time needed for running. These findings indicated that the model proposed in this study was advantageous over the deep learning $\mathrm{CNN}$ models in any of the investigated indices: it better extracted the features of ancient murals with a more powerful generalization capacity and more stable classification outcomes.

\section{(3) Accuracy in dynasty recognition}

After 10,000 running steps, the accuracy rate of the proposed model in dynasty recognition was compared with those of the modified CNNs in the literature [31-34]. The results are summarized in Table 5.

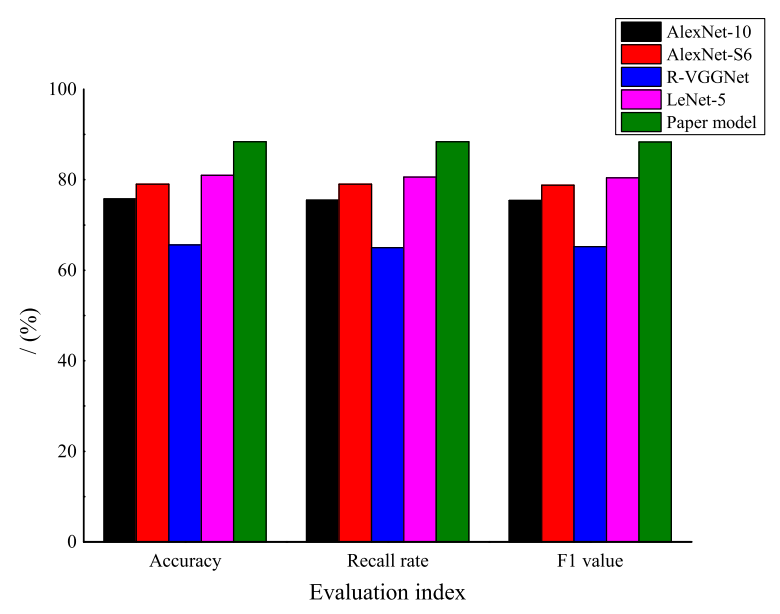

Fig. 10 Comparison of the evaluation indices among different models 
Table 5 Comparison of the accuracy rates of different models for different categories of murals

\begin{tabular}{llllllll}
\hline & $\begin{array}{l}\text { Stone } \\
\text { Age }\end{array}$ & $\begin{array}{l}\text { Qin/Han } \\
\text { Dynasty }\end{array}$ & $\begin{array}{l}\text { Sui/Tang } \\
\text { Dynasty }\end{array}$ & $\begin{array}{l}\text { Song/Jin } \\
\text { Period }\end{array}$ & $\begin{array}{l}\text { Weijin } \\
\text { Period }\end{array}$ & $\begin{array}{l}\text { Ming/Qing } \\
\text { Dynasty }\end{array}$ & Average \\
\hline $\begin{array}{l}\text { Literature } \\
{[25]}\end{array}$ & 93.23 & 80.36 & 75.43 & 79.86 & 86.32 & 80.46 & 82.61 \\
$\begin{array}{l}\text { Literature } \\
{[26]}\end{array}$ & 90.45 & 82.70 & 79.54 & 76.50 & 88.53 & 76.45 & 82.36 \\
$\begin{array}{l}\text { Literature } \\
{[27]}\end{array}$ & 93.57 & 79.76 & 77.87 & $\mathbf{8 6 . 1 2}$ & 89.60 & $\mathbf{8 5 . 3 0}$ & 85.37 \\
$\begin{array}{l}\text { Literature } \\
{[28]}\end{array}$ & $\mathbf{9 8 . 9 5}$ & 83.21 & 80.58 & 78.8 & 85.65 & 80.39 & 83.26 \\
$\begin{array}{l}\text { Paper } \\
\text { model }\end{array}$ & 97.50 & $\mathbf{8 5 . 8 2}$ & $\mathbf{8 5 . 6 7}$ & 83.80 & $\mathbf{9 2 . 6 4}$ & 83.89 & 88.4 \\
\hline
\end{tabular}

Note: The bold numbers represent the highest classification accuracy rates

As shown in Table 5, compared with other CNNs, the model proposed in this study achieved an average increase in the accuracy rate of $7.42 \%$ for the recognition of most categories. All methods achieved a rather high accuracy rate for the murals created in the Stone Age because most murals in this period were created on rocks or walls and their contents were primarily animals. Compared with the murals created in other periods, those in the Stone Age are feature-contrasted. From the Qin/Han dynasty and Sui/Tang dynasty to the Song/Jin period, the contents gradually changed from people, palaces, and temple murals and sutra murals to tomb murals. Due to the similarities in color, clothing, buildings, and painting styles among these murals, feature extraction for dynasty recognition became difficult.

\section{(4) Comparisons with DenseNet and MobileNet}

Model complexity has a great influence on the running time and classification accuracy of the model. Specifically, time complexity influences the duration of the training time of the model, while spatial complexity influences the number of model parameters: excessive time complexity will cause excessive time waste during model training and classification prediction, which affects the prediction and training speeds of the model; a high spatial complexity will cause overabundant parameters, which is likely to cause overfitting during training. To verify the satisfactory performance of the proposed model in terms of both time complexity and spatial complexity, we compared its complexities and classification accuracy with those of two other deep learning models, i.e.,

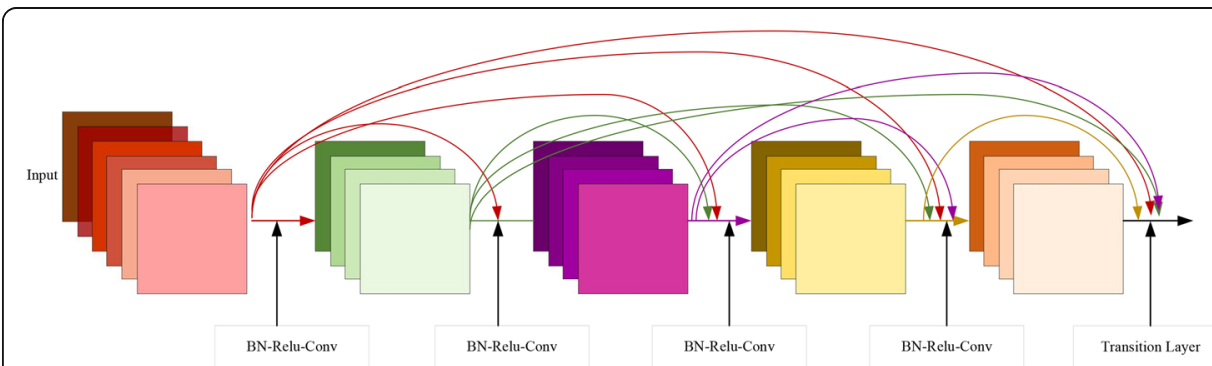

Fig. 11 DenseNet network structure diagram 
Table 6 MobileNet network structure

\begin{tabular}{|c|c|c|}
\hline Type/stride & Filter shape & Input size \\
\hline Conv/s2 & $3 \times 3 \times 3 \times 32$ & $224 \times 224 \times 3$ \\
\hline Conv dw/s1 & $3 \times 3 \times 32 d w$ & $112 \times 112 \times 32$ \\
\hline Conv/s1 & $1 \times 1 \times 32 \times 64$ & $112 \times 112 \times 32$ \\
\hline Conv dw/s2 & $3 \times 3 \times 64 d w$ & $112 \times 112 \times 64$ \\
\hline Conv/s1 & $1 \times 1 \times 64 \times 128$ & $56 \times 56 \times 64$ \\
\hline Conv dw/s1 & $3 \times 3 \times 128 d w$ & $56 \times 56 \times 128$ \\
\hline Conv/s1 & $1 \times 1 \times 128 \times 128$ & $56 \times 56 \times 128$ \\
\hline Conv dw/s2 & $3 \times 3 \times 128 d w$ & $56 \times 56 \times 128$ \\
\hline Conv/s1 & $1 \times 1 \times 128 \times 256$ & $28 \times 28 \times 128$ \\
\hline Conv dw/s1 & $3 \times 3 \times 256 d w$ & $28 \times 28 \times 256$ \\
\hline Conv/S1 & $1 \times 1 \times 256 \times 256$ & $28 \times 28 \times 256$ \\
\hline Conv dw/s2 & $3 \times 3 \times 256 d w$ & $28 \times 28 \times 256$ \\
\hline Conv/s1 & $1 \times 1 \times 256 \times 512$ & $14 \times 14 \times 256$ \\
\hline $\begin{array}{l}\text { Conv dw/s1 } \\
\text { Conv/s1 }\end{array}$ & $\begin{array}{l}3 \times 3 \times 512 d w \\
1 \times 1 \times 512 \times 512\end{array}$ & $\begin{array}{l}14 \times 14 \times 512 \\
14 \times 14 \times 512\end{array}$ \\
\hline Conv dw/s2 & $3 \times 3 \times 512 d w$ & $14 \times 14 \times 512$ \\
\hline Conv/s1 & $1 \times 1 \times 512 \times 1024$ & $7 \times 7 \times 512$ \\
\hline Conv dw/s2 & $3 \times 3 \times 1024 d w$ & $7 \times 7 \times 1024$ \\
\hline Conv/S1 & $1 \times 1 \times 1024 \times 1024$ & $7 \times 7 \times 1024$ \\
\hline Avg Pool/S1 & Poop $7 \times 7$ & $7 \times 7 \times 1024$ \\
\hline $\mathrm{FC} / \mathrm{s} 1$ & $1024 \times 1000$ & $1 \times 1 \times 1024$ \\
\hline Softmax/s1 & Classifier & $1 \times 1 \times 1000$ \\
\hline
\end{tabular}

DenseNet and MobileNet, over the mural datasets. The network structure of DenseNet is shown in Fig. 11, and that of MobileNet is summarized in Table 6.

DenseNet possesses a deep network structure. Compared with ResNet, DenseNet has fewer parameters. During training, the insertion of a BN layer can achieve a certain regularization effect. DenseNet contains multiple dense blocks for downsampling. Compared with existing networks, DenseNet can receive a small number of feature maps as model outputs; it reuses features from the feature maps of different network layers and, thus, avoids the problems of gradient loss and model reduction. MobileNet is a convolutional neural network that is constructed by isolating the convolution from the two aspects of space and channels. It has the merits of a light weight and deep structure. Both MobileNet and Inception-v3 adopt convolution separation to enhance the calculation efficiency of the model.

The accuracy rates, calculation amounts, and parameter numbers of the proposed model, DenseNet and MobileNet are summarized in Table 7. Compared with DenseNet and Inception-v3, MobileNet has fewer parameters and requires less memory.

Table 7 Comparison of the accuracies and complexities of the three models

\begin{tabular}{llllll}
\hline & Input size & Calculated & Parameter & Accuracy & Memory rate \\
\hline DenseNet & $224 \times 224 \times 3$ & 2.91 GFLops & 40 Million & $79.8 \%$ & $200 \mathrm{MB} \sim 500 \mathrm{MB}$ \\
MobileNet & $224 \times 224 \times 3$ & 0.5 GFLops & 4.2 Million & $82.5 \%$ & $74 \mathrm{MB}$ \\
Inception-v3 & $299 \times 299 \times 3$ & 1.6 GFLops & 23.5 Million & $88.4 \%$ & $90 \mathrm{MB} \sim 100 \mathrm{MB}$ \\
\hline
\end{tabular}


DenseNet has the most parameters, but its accuracy is lowest due to the limitation of the experimental hardware platform. The primary reason is that the large parameter number of DenseNet increases its demand for memory during training and thus slows the training process. During training, the deep network level of DenseNet and the dense connection between two networks increase the memory requirement. During backpropagation, features are continuously input from the previous layer to the next layer due to repeated reuse of the features, which causes feature overstock. With such a condition, explosion due to overabundant features is likely to occur. MobileNet has the smallest number of parameters, which can accelerate the calculation speed. However, the adoption of the rectified linear unit (ReLU) activation function in MobileNet causes neuronal silencing. For the input mural images, the ReLU activation function can cause the loss of some information during feature transformation. In this study, we employed two ReLU activation functions between Inception modules and an ALR-SGD algorithm, which increased the representativeness of the model. In addition, because both DenseNet and MobileNet belong to deep networks, they need longer training times with a slower convergence speed. In this study, the fusion of transfer learning accelerated the convergence of the model and its training time.

\subsection{Comparisons of different datasets}

To further validate the recognition precision of the transfer learning-fused Inception-v3 model constructed in this study for ancient murals, it was trained on other public datasets, and the results were compared.

(1) WikiArt [35]. WikiArt is a dataset based on artists and their arts, that is, each category contains art created by the same artist. In this study, a total of 17,386 paintings by 17 artists, whose styles varied, were utilized. The paintings were divided into a training set, testing set, and validation set according to the

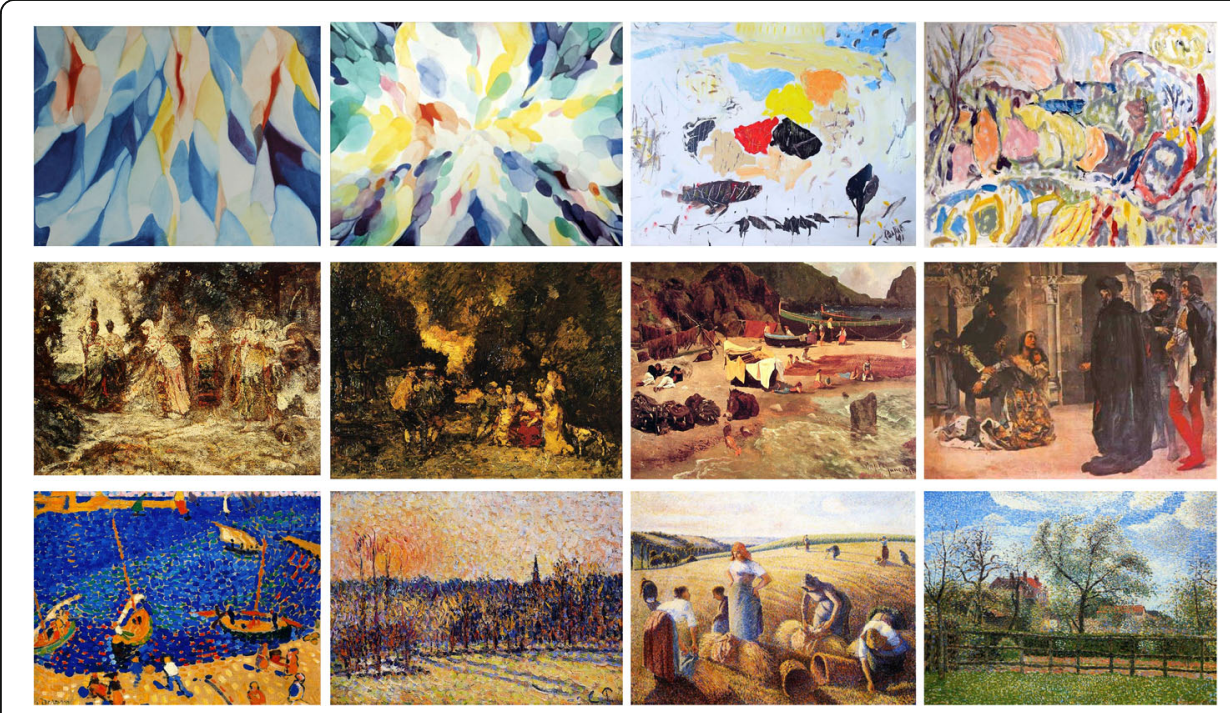

Fig. 12 Some examples of the drawing images 

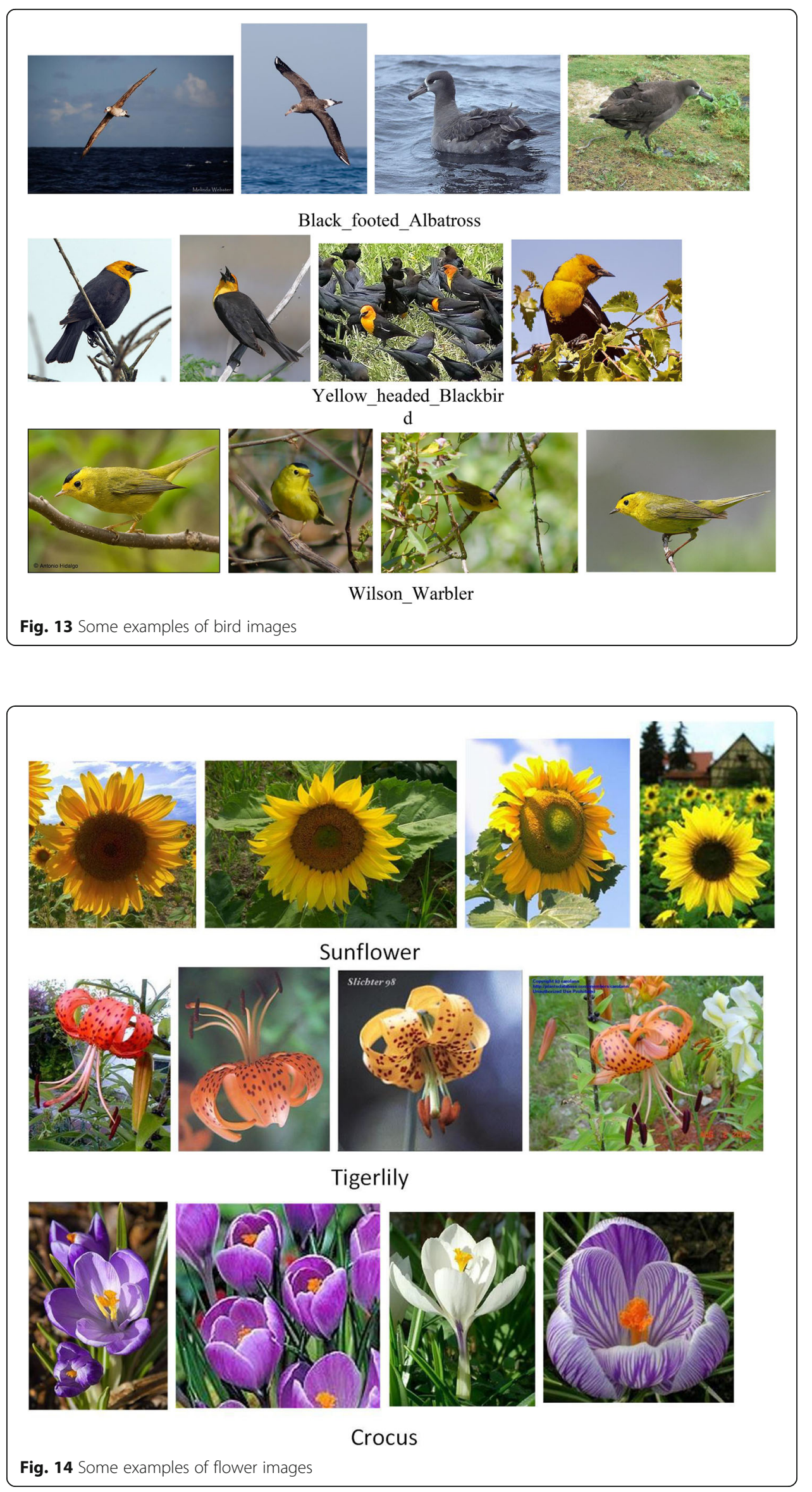
proportions of $80 \%, 10 \%$, and $10 \%$, respectively. Examples of these images are provided in Fig. 12.

(2) Caltech-UCSD Birds [36]. Caltech-UCSD Birds is a dataset that contains bird images; it contains 200 different kinds of birds with a total of 11,788 images. In this study, 50 different kinds of birds were selected from the dataset, and the images were divided into a training set, testing set, and validation set according to proportions of $80 \%$, 10\%, and 10\%, respectively. Examples of the bird images are provided in Fig. 13.

(3) 17_Category_Flower_Dataset [37]. This dataset includes 17 kinds of flowers, and each category contains 80 images, with 1360 images in total. In this study, all images in the dataset were employed for the validation experiment. Furthermore, 64 images were used for training, 8 images were employed for testing, and 8 images were utilized for validation. Examples of some categories of flowers are given in Fig. 14.

(4) DH660 [38]. In this study, the Dunhuang Flying Apsaras images created in 10 dynasties contained in DH660 were selected, with a total number of 610 images. They were divided into the training set, testing set, and validation set according to proportions of $80 \%, 10 \%$, and 10\%, respectively. Examples of the Flying Apsaras images are provided in Fig. 15.

Under the conditions of 1000 running steps with a learning rate of 0.1 , the accuracy rates of the proposed model for different datasets are summarized in Table 8 .

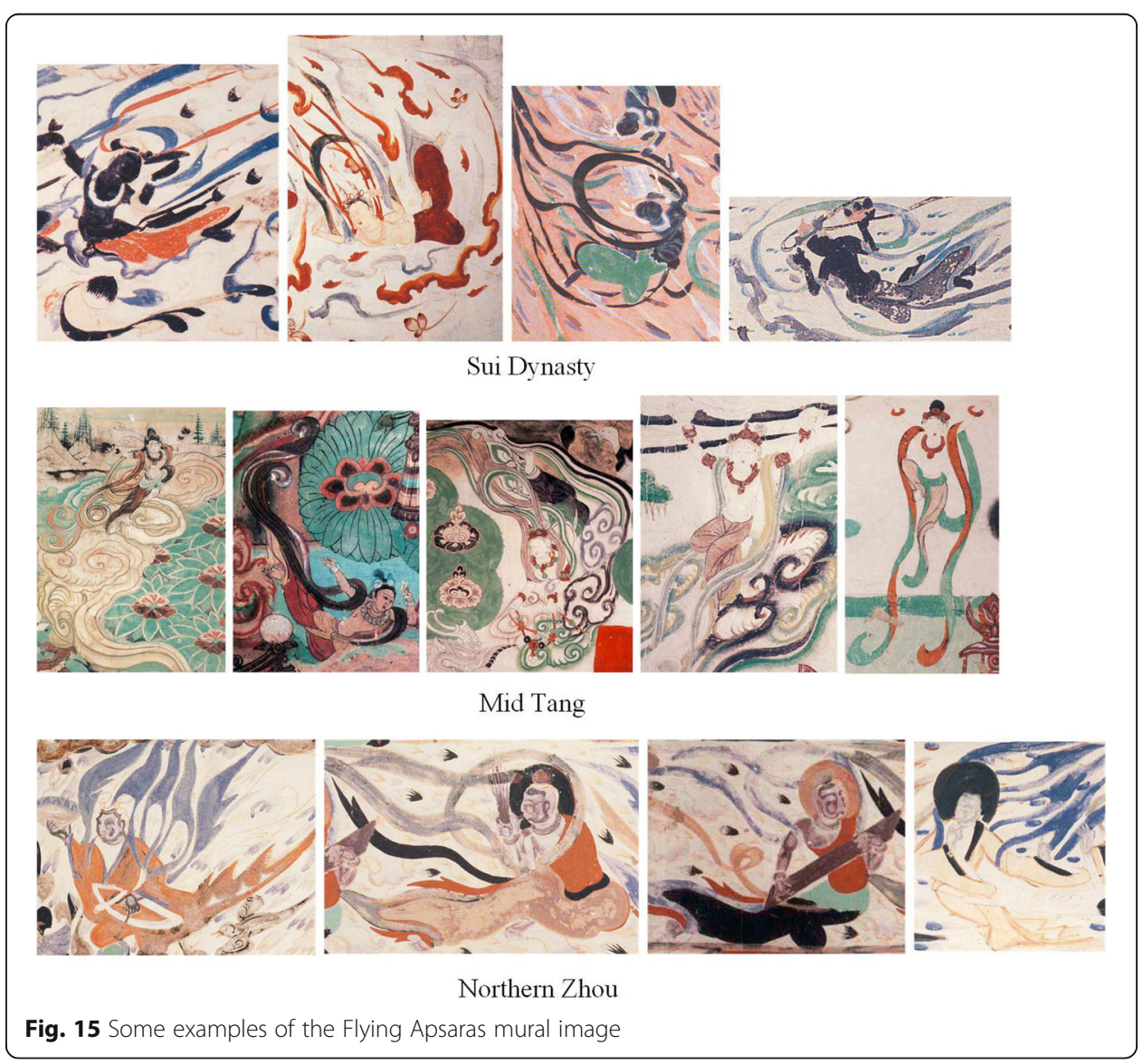


Table 8 Accuracy of the model for each dataset

\begin{tabular}{ll}
\hline Dataset name & Accuracy/\% \\
\hline WikiArt & 53.5 \\
Caltech-UCSD Birds & 76.0 \\
17_Category_Flower_Dataset & 94.7 \\
DH660 & 88.3 \\
\hline
\end{tabular}

As shown in Table 8, the accuracy rates of the proposed model on WikiArt, CaltechUCSD Birds, 17_Category_Flower_Dataset, and DH660 were 53.5\%, 76.0\%, 94.7\%, and 88.3\%, respectively. These accuracy rates were compared; the results are shown in Fig. 16.

As shown in Fig. 14, the proposed model exhibited relative stability during the whole training process, without wide-ranging fluctuations. The model obtained the highest accuracy for the 17_Category_Flower_Dataset because this dataset contains only 1360 images, which is a small number compared with the 9700 mural images constructed in this study. Therefore, the proposed model could easily extract the features of natural flower images, thereby achieving a high classification accuracy rate. The accuracy rate of the proposed model for the WikiArt painting dataset was the lowest. Compared with WikiArt and Caltech-UCSD Birds, its accuracy for the mural dataset was higher, which indicates that the performance of the model proposed in this study is stable on mural image datasets and is suitable for the dynasty-based classification of ancient murals.

Based on these experimental results, the model proposed in this study achieved higher accuracy for recognizing dynasties when ancient murals were created than other classical and modified CNNs and achieved better performance on the mural image dataset constructed in this study. However, there were also misclassifications; some of these misclassifications are provided in Figs. 17, 18, 19, and 20.

- Misclassification 1: due to the mural content. In Fig. 17, both images contain the carriage, horse and Buddha, and the model extracted similar features.

- Misclassification 2: due to similar mural color and painting styles. Figure 18 shows two murals that were created in the Song/Jin period, and Fig. 19 shows murals

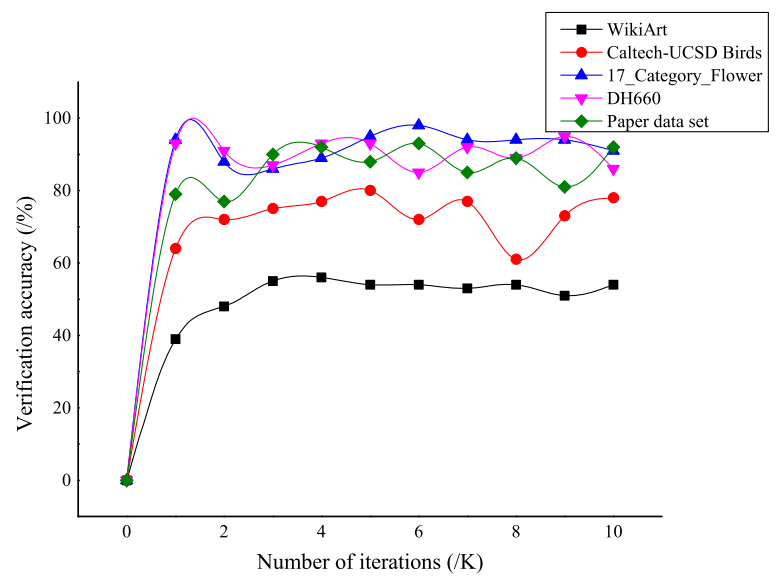

Fig. 16 Comparison of experimental accuracy among different datasets 


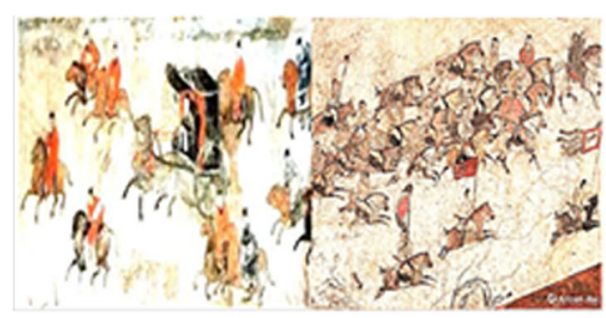

(a)

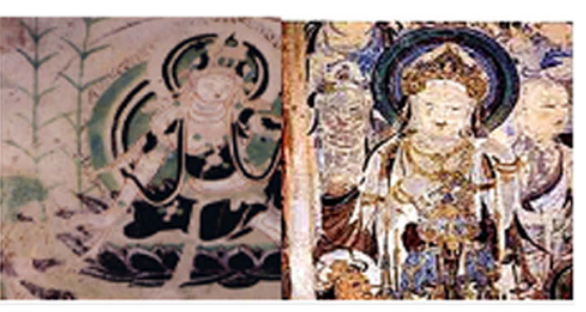

(b)

Fig. 17 Example of misclassification of similar mural content. a The mural created in the Qin/Han Dynasties is misclassified as the Sui/Tang Period (left) and that created in the Sui/Tang Period is misclassified as the Qin/Han Dynasties (right). b The mural created in the Song/Jin Period is misclassified as the Sui/Tang Period (left) and that created in the Sui/Tang Period is misclassified as the Song/Jin Period (right)

created in the Wei/Jin dynasties. Because all murals in these figures were obtained from the Dunhuang Caves and are similar in color and painting styles, misclassifications tend to occur.

- Misclassification 3: due to large-area shedding. In Fig. 20, misclassification occurred for all images. The reason is that all murals were subjected to multiple sheddings, which causes difficulty in mural feature extraction.

These cases represented failed classification cases. The main reason for these misclassifications is that the elements of murals, such as Bodhisattvas, figures, animals, and buildings, were drawn directly on the wall with a paintbrush in different dynasties. Influenced by the cultural spread, religious transmission, and living customs, different murals in different grottoes may bear similarities in mural color and figure lines, which makes it difficult to identify dynasties according to the extracted features (e.g., color and lines). In addition, all mural images have transcended a long historical time. The pigments on the image and wall are eroded by pests, natural disasters, and pathological changes, which make the mural image partially shed. The characteristics of murals with a large shedding-off area lack integrity, which impedes dynasty recognition.

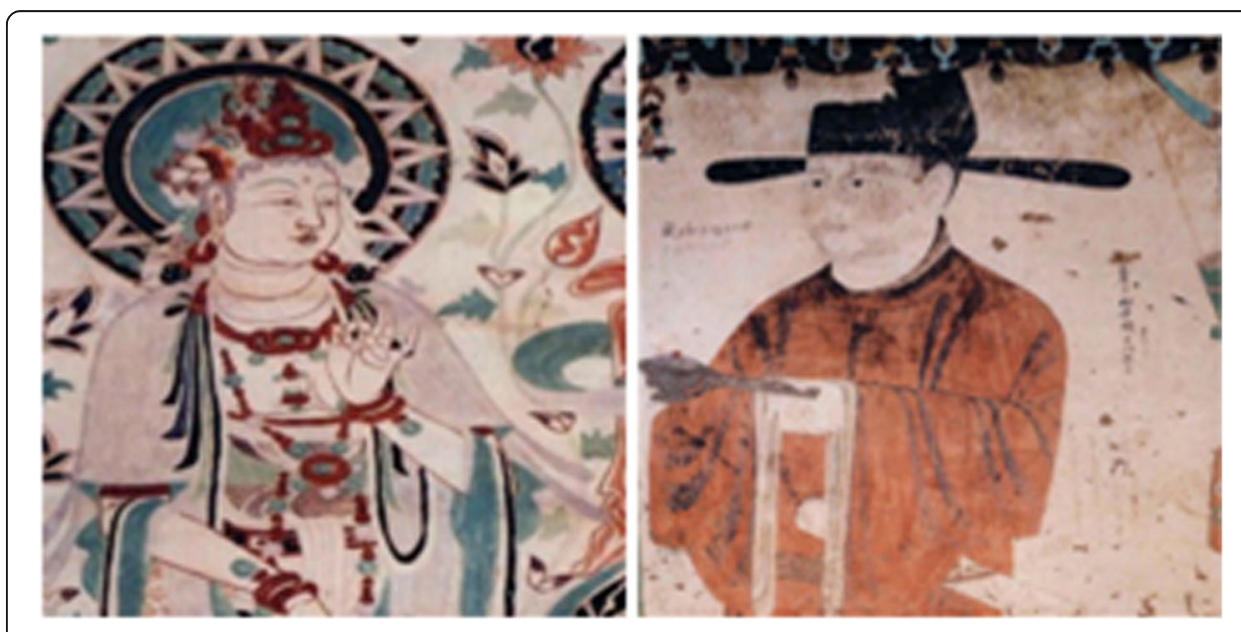

Fig. 18 Mural images of the Song/Jin dynasties 

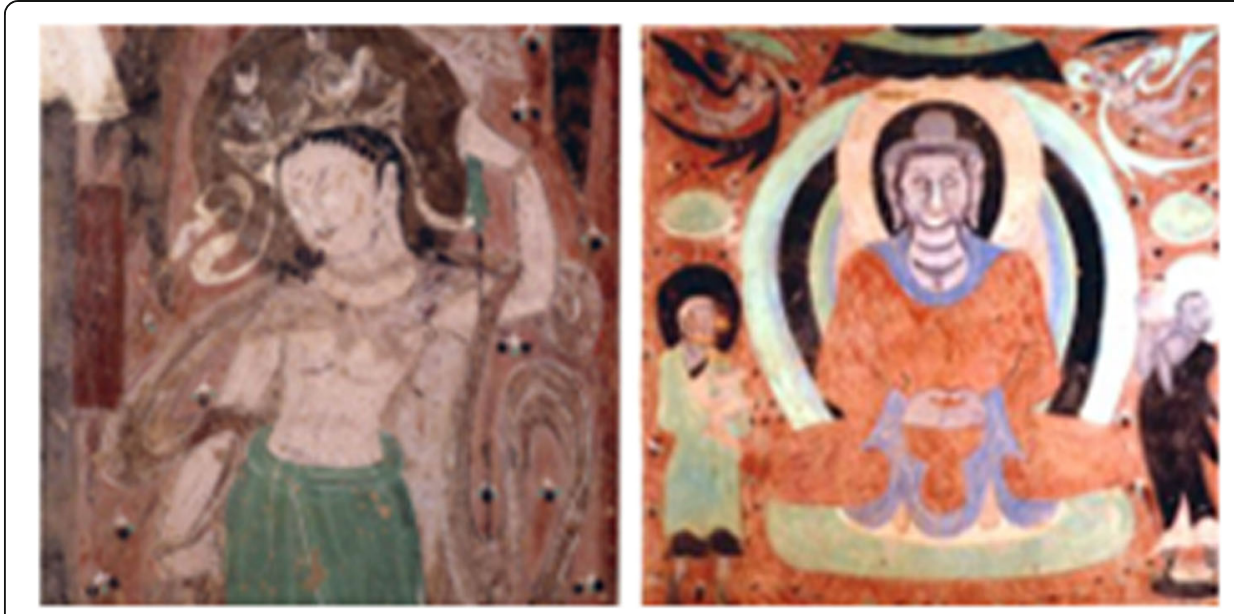

Fig. 19 Frescoes of the Wei and Jin dynasties

\section{Conclusions}

Targeting the problems of low dynasty-based classification accuracy and difficult feature extraction for ancient mural images as well as similarities in the contents and painting styles of mural images, this study proposed a transfer learning-fused Inception-v3 model for dynasty-based classification of ancient murals. Due to the difficulty of the selection of mural datasets, we expanded the datasets available with a data enhancement algorithm. The results showed that the proposed model achieved a classification accuracy rate of $88.4 \%$ for the testing dataset and shortened the running time. Compared with the classic deep learning models and modified CNNs, the model proposed in this study exhibited better performance and higher accuracy, reaching 10,000 steps within a short time. Furthermore, its calculation burden was small, with a low cost and a small occupied memory space.

When the recall rate and F1 score were introduced for evaluation, the proposed model exhibited more satisfactory classification accuracy, with a recall rate of $88.36 \%$ and an F1 score of $88.32 \%$, which increased by $8 \%$ on average compared with other models. After training and testing on WikiArt, Caltech-UCSD Birds, 17_Category_Flower_Dataset, and DH660, there was no noticeable difference in the recognition accuracy of the model for DH660 and for the constructed ancient mural dataset, which indicates that the model proposed in this study is suitable for dynasty recognition of ancient mural images.

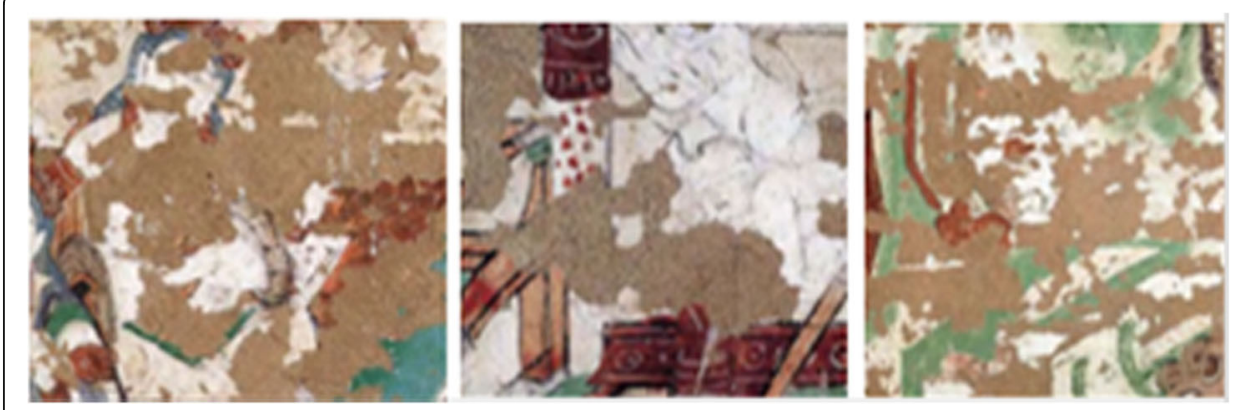

Fig. 20 Murals with large areas of image shedding 
In the experiment, due to the hardware environment and the small difference in the painting styles of some ancient mural images, there were some shortcomings in using the classical depth network model to train the dataset in this study. For instance, the accuracy rate was not high due to the rich features of mural images that could not be extracted more deeply, and misclassifications sometimes occurred. In the future, the datasets need to be further expanded, and the network structure needs to be adjusted to extract more in-depth features of mural image texture and color. In addition, attempts to modify other models can be made in further research on the dynasty classification of ancient murals, with the expectations of improving the classification accuracy of ancient murals and realizing rapid and effective dynasty recognition of ancient murals.

\section{Abbreviations}

CNN: Convolutional neural network; SIFT: Scale-invariant feature transform; BN: Batch normalization; FC: Fully connected; ALR-SGD: Adaptive learning rate on stochastic gradient descent

\section{Acknowledgements \\ None.}

\section{Authors' contributions}

JC devised the study plan and led the writing of the article. MY, YJa, and XT conducted the experiment and collected the data. ZZ conducted the analysis, and JC supervised the whole process. All authors read and approved the final manuscript.

\section{Funding}

This study was supported by the Natural Science Foundation of Shanxi Province (201701D21059), Key Research Base Project of Humanities and Social Sciences in Colleges and University of Shanxi Province (20190130), Art and Science Planning Project of Shanxi Province (2017F06), and Xinzhou Platform and Talent Project (20180601).

The funding organizations had no role in the design of the study, the collection, analysis, and interpretation of data or the writing of the manuscript.

\section{Availability of data and materials}

All data generated or analyzed during this study are included in this article.

\section{Declarations}

\section{Competing interests}

The authors declare that they have no competing interests.

Received: 13 October 2020 Accepted: 1 June 2021

Published online: 27 July 2021

\section{References}

1. X.J. Kang, Conservation of a silk robe excavated from the Loulan Mural Tomb, Xinjiang, China. Stud. Conserv. 59(S1), S55-S57 (2014)

2. G.K. Wang, H.Q. Wang, Y. Yin, et al., Color restoration and evaluation of murals based on spectral reconstruction technique. Opt. Laser Technol. 43(02), 280-285 (2019)

3. Y.P. Pan, D.M. Lu, Digital protection and restoration of ancient Dunhuang frescoes. J. Syst. Simul. 15(3), 310-314 (2003)

4. Zeng Y, Gong Y, Nearest neighbor based digital restoration of damaged ancient Chinese paintings. Proc of the 2018 IEEE 23rd International Conference on Digital Signal Processing (IEEE, Shanghai, China, 2018), p. 19-21.

5. H. Wang, Q.Q. Li, Q. Zou, Inpainting of dunhuang murals by sparsely modeling the texture similarity and structure continuity. J. Comput. Cult. Herit. 12(3) (2019)

6. H.J. Zhang, S. Wang, X.F. Xu, et al., Tree2Vector: learning a vectorial representation for tree-structured data. IEEE Trans. Neural Netw. Learn. Syst. 29(11), 5304-5318 (2018)

7. D.W. Tang, D.M. Lu, D.Q. Xu, Grouping multi-Instance learning method in mural image classification. Chin. J. Image Graph. 19(5), 708-715 (2014)

8. Q. Wang, D.M. Lu, Composition analysis-based relevance ranking for ancient mural. J. Zhejiang. Univ. Eng. Sci. 46(3), 392-401 (2012)

9. D.W. Tang, D.M. Lu, B. Yang, et al., Measurement of mural image similarity under constraint of overall structure of contour. Chin. J. Image Graph. 18(8), 968-975 (2013)

10. Y.N. Wang, D.N. Zhu, H.Q. Wang, et al., Classification of paint multi-spectral images in mural paintings based on convolutional neural network. Adv. Laser Optoelectronics 56(22), 48-56 (2019)

11. Sun F, Study on image data classification of Taoist murals in the Ming and Qing Dynasties in Northern Shaanxi. 2019, 2(10).

12. B. Hao, Research and implementation of the classification algorithm of Chinese ancient character murals based on style characteristics (Tianjin University, Tianjin, 2017) 
13. L.J. Zhou, Q.W. Li, G.Y. Huo, Y. Zhou, Image classification using biomimetic pattern recognition with convolutional neural networks features. Computa. Intell. Neurosci. 2017, 3792805 (2017)

14. P. Tang, X.G. Wang, B.G. Shi, X. Bai, Deep FisherNet for image classification. IEEE Trans. Neural Netw. Learn. Syst. 99, 1-7 (2018)

15. Z.L. Hao, X.S. Luo, S.L. Zhao, Single-cell image classification based on same-layer multi-scale kernel CNN. Comput. Eng. Appl. 54(15), 181-184 (2018)

16. Q.Q. Li, Q. Zou, D. Ma, Q. Wang, S. Wang, Dating ancient paintings of Mogao Grottoes using deeply learnt visual code. Sci. China Inf. Sci. 2018, 092105 (2018)

17. Q. Zou, Y. Cao, Q.Q. Li, C.H. Huang, S. Wang, Chronological classification of ancient paintings using appearance and shape features. Pattern Recognit. Lett. 49, 146-154 (2014)

18. Szegedy C, Vanhoucke V, loffe S, Shlens J, Rethinking the inception architecture for computer vision. Proc of the IEEE Conference on Computer Vision and Pattern Recognition (IEEE, Las Vegas, USA, 2016), p. 2818-2826.

19. Z. Liu, C. Yang, J. Huang, et al., Deep learning framework based on integration of S-Mask R-CNN and Inception-v3 for ultrasound image-aided diagnosis of prostate cancer. Future Generation Comput. Syst. 114, 358-367 (2020)

20. Chowdary MK, Nguyen TN, Hemanth DJ, Deep learning-based facial emotion recognition for human-computer interaction applications. Neural Comput. Appl. (2021). https://doi.org/https://doi.org/10.1007/s00521-021-06012-8.

21. C.H. Yeh, C.H. Lin, L.W. Kang, et al., Lightweight deep neural network for joint learning of underwater object detection and color conversion. IEEE Trans. Neural Netw. Learn. Syst. (2021)

22. X.B. Shi, X.J. Pang, D.Y. Zhang, Z. Guo, Image classification based on deep learning mixed model transfer learning. J. Syst. Simul. 28(01), 167-173 + 182 (2016)

23. Krizhevsky A, Sutskever I, Hinton GE, ImageNet classification with deep convolutional neural networks. Proceedings of the 25th International Conference on Neural Information Processing Systems (Curran Associates Inc, Lake Tahoe, Nevada, 2012), p. 1097-1105.

24. He KM, Zhang XY, Ren SQ, Sun J, Deep residual learning for image recognition. Proc of the IEEE Conference on Computer Vision and Pattern Recognition (IEEE, Las Vegas, USA, 2016), p. 770-778.

25. Simonyan K, Zisserman A, Very deep convolutional networks for largescale image recognition [EB/OL], 2014. 20140924 [20150410. https://arxiv.org/pdf/1409.1556/.pdf.

26. Huang G, Liu Z, Laurens V, et al, Densely connected convolutional networks, IEEE Computer Society. IEEE Computer Society (2016).

27. Howard AG, Zhu M, Chen B, et al, MobileNets: efficient convolutional neural networks for mobile vision applications (2017).

28. Zhang RM, Zhang $\mathrm{Q}$, Chen B, A license plate recognition algorithm based on improved Lenet-5. Science Technology and Engineering 20(12), 4775-4779 (2020).

29. Xiao LS, Yan Q, Deng SY, Scene classification with improved AlexNet model. 12th International Conference on Intelligent Systems and Knowledge Engineering (IEEE, Nanjing, China, 2017), p. 1-6.

30. Xu ZJ, Wang Y. Glaucoma fundus images classification method based on transfer learning. Computer Engineering and Applications 57(03), 144-149 (2021).

31. Z.Q. Zhong, J. Yuan, X.Y. Tang, Left and right eye recognition based on convolutional neural network. Comput. Res. Dev. 55(08), 1667-1673 (2018)

32. Y. Du, R. Zhang, A. Zargari, T.C. Thai, C.C. Gunderson, K.M. Moxley, H. Liu, B. Zheng, Y.C. Qiu, Classification of tumor epithelium and stroma by exploiting image features learned by deep convolutional neural networks. Annals of Biomedical Engineering 46(12), 1988-1999 (2018)

33. O. Eminaga, N. Eminaga, A. Semjonow, B. Breil, Diagnostic classification of cystoscopic images using deep convolutional neural networks. JCO Clin. Cancer Inform. 17(2), 1232 (2018)

34. G. Wang, M. Lü, T. Li, G.L. Yuan, W.Z. Liu, Convolutional neural network based on spatial pyramid for image classification. J. Beijing Inst. Technol. 27(04), 630-636 (2018)

35. WikiArt, WikiArt the online home for visual arts from all around the world, 2016.

36. Wah C, Branson S, Weinder P, The caltech-ucsd birds-200-2011 dataset, 2011.

37. M.E. Nilsback, Z. Andrew, A visual vocabulary for flower classification. Proc. IEEE Conf. Comput. Vision Pattern Recognit. 2, 1447-1454 (2006)

38. Q. Zou, L.H. Ni, Q. Wang, Z.W. Hu, Q.Q. Li, S. Wang, Local pattern collocations using regional co-occurrence factorization. IEEE Trans. Multimedia 19, 492-505 (2017)

\section{Publisher's Note}

Springer Nature remains neutral with regard to jurisdictional claims in published maps and institutional affiliations. 